\title{
Two-particle correlation via Bremsstrahlung
}

\author{
Soyeon Cho ${ }^{1, a}$, Kayoung Park ${ }^{1}$, and Jin-Hee Yoon ${ }^{1, b}$ \\ ${ }^{1}$ Department of Physics, Inha University, Incheon, Republic of Korea
}

\begin{abstract}
Ridge is the well-known structure in two-particle angular correlations at highenergy heavy-ion collisions. This structure is physically understood through elliptic and higher-order flows at nucleus-nucleus collisions. This behavior is also found in small systems, such as proton-proton collisions, recently. However, Ridge structure in small system is hard to be understood using hydrodynamics, since small systems are not dense enough to produce the Quark-Gluon plasma. Thus, we try to describe this phenomena through kinematic interaction between jets and medium partons. In high-energy heavyion collision, the energetic particles called jets go out in specific direction and lose their energy while passing through the medium. During such process, photons/gluons are emitted from interaction between jets and medium partons. We concentrate on energy loss via photon radiations, known as Bremsstrahlung. Recently, two symmetric double scattering processes between jet particle and medium parton are reported to be able to produce certain constructive interference, which gives collective motion and medium partons are aligned along incoming jet particles. We conjecture that similar behavior might happen in Bremsstrahlung processes, and therefore we consider the two symmetric diagrams of photon emission and medium parton scattering. We expect these two amplitudes to give constructive interference leading to the collective motion of medium. We check the correlation between emitted photon and final jet, and those between medium parton and final jet for high-energy jet. To describe parton momentum distribution in medium, we use the Maxwell-Boltzmann distribution. We discover collective motion in both angular correlations. We also check the tendency of the angular correlation for two particles according to the incident angle of jet particle, energy of emitted photon and temperature of systems, respectively. We can conclude that collective motion is able to be understood through kinematic description.
\end{abstract}

\section{Introduction}

Ridge structure which is pseudo-rapidity independent shape is found in two-particle angular correlation for high-energy heavy-ion collisions. This structure can be explained by hydrodynamics, especially elliptic and higer-order flows. Recently, Ridge structure is also discovered in small systems such as proton-proton collisions with high energies. Such case is also understood through hydrodynamics but many physicist do not expect that hot and dense medium (Quark-Gluon plasma state) is produced at small systems, hence it should be understood carefully. Thus we try to describe Ridge structure through kinematic interaction between jets and medium, such as energy-loss mechanism.

\footnotetext{
a e-mail: soyeon.cho@cern.ch

be-mail: jinyoon@inha.ac.kr
} 


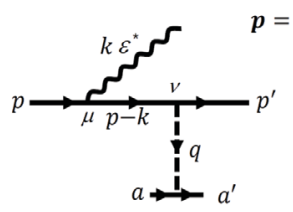

(a)

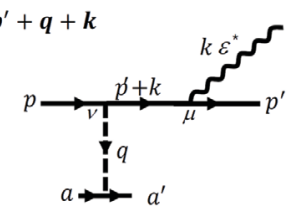

(b)
Figure 1. Two symmetric Feynman diagrams for Bremsstrahlung process.

In high-energy heavy-ion collisions, high-energy particles which come out in a particular direction are called as jet. When jets pass through the medium, they lose their partial energy by interacting medium partons. The main interaction is gluon or photon radiation process, which gluons or photons are emitted by scattering between jet particles and medium partons. In our study, we attempt to produce Ridge structure from energy-loss mechanism of jets by emitting gluons or phtons. First of all, we consider only phton radiation process, known as Bremsstrahlung.

\section{Formalism}

Wong reported that certain constructive interference appears in two symmetric double scattering processes between jet particle and medium parton. [4] This interference produces collective motion and medium partons are aligned along incident jet particles.

In our study, we conjecture that such constructive interference also arises in the Bremsstrahlung process. Thus, we consider two symmetric diagrams of $\gamma$ emission and medium parton scattering as shown as Figure 1. We expect these two amplitudes give constructive interference leading to collective motion for medium, since the cross section is the absolute-square of the sum of amplitudes, as in equation (1).

$$
d \sigma \sim\left|M_{(a)}+M_{(b)}\right|^{2}=\left|M_{(a)}\right|^{2}+\left|M_{(b)}\right|^{2}+(\text { interference })
$$

Here each amplitudes are given as

$$
\begin{gathered}
M_{(a)}=-i \bar{u}\left(p^{\prime}\right)\left(-i g \gamma^{v}\right) \frac{1}{q^{2}} \bar{u}\left(a^{\prime}\right)\left(-i g \gamma_{v}\right) u(a) \frac{i(\not p-\not k+m)}{(p-k)^{2}-m^{2}+i \epsilon}\left(-i g \gamma^{\mu}\right) \epsilon_{\mu}^{*} u(p) \\
M_{(b)}=-i \bar{u}\left(p^{\prime}\right) \epsilon_{\mu}^{*}\left(-i g \gamma^{\mu}\right) \frac{i\left(p^{\prime}+\not k+m\right)}{\left(p^{\prime}+k\right)^{2}-m^{2}+i \epsilon}\left(-i g \gamma^{v}\right) \frac{1}{q^{2}} \bar{u}\left(a^{\prime}\right)\left(-i g \gamma_{v}\right) u(a) u(p) .
\end{gathered}
$$

\section{Results}

We calculate the angular correlation for final jet particle and emitted photon and that for final jet particle and final medium parton. We check the tendency of the angular correlation for two particles according to the incident angle of jet particle, energy of emitted photon and temperature of systems, separately. We set the outgoing direction of jet particle along $z$-axis and incoming and outgoing jets are on $x-z$ plane. We set the initial and final energy of incoming jet particle to be $10 \mathrm{GeV}$ and $9 \mathrm{GeV}$. To describe momentum distribution of initial medium partons, we apply Maxwell-Boltzmann distribution function. We also confirmed that results are not changed significantly when we use FermiDirac distribution function instead of Maxwell-Boltzmann distribution function.

The Figure 2 shows angular correlation between final jet particle and emitted photon for various incident angles of jet particle $\theta_{p}$, energies of emitted photon $E_{k}$ and temperatures of system $k_{B} T$. We 

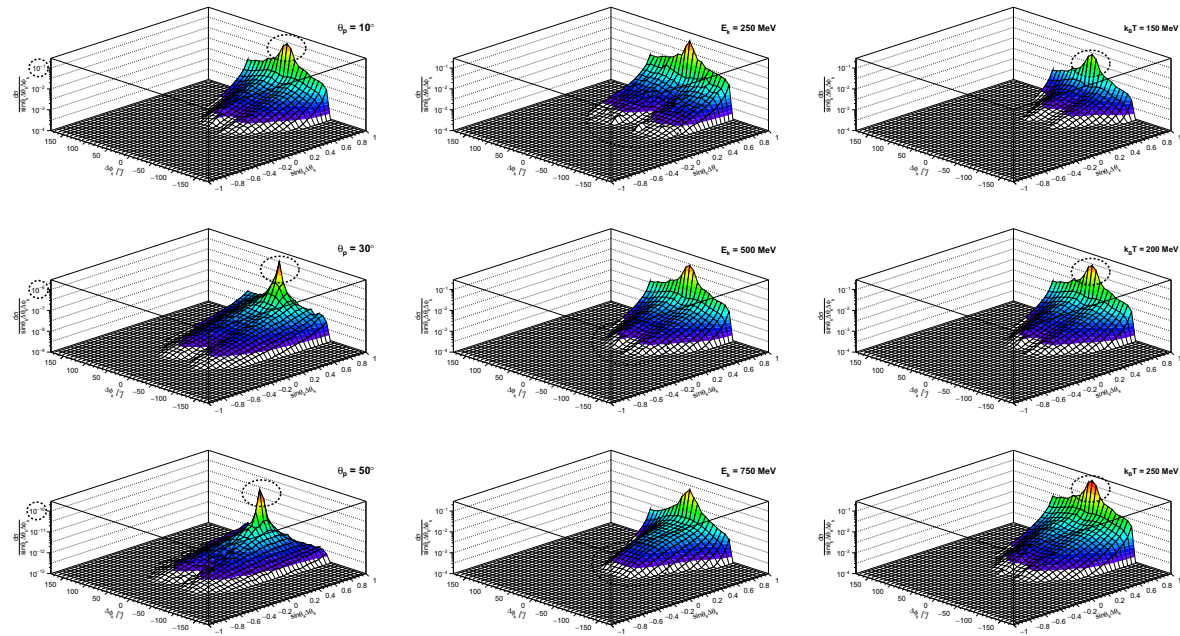

Figure 2. Angular correlation between final jet particle and emitted phton.

change $\theta_{p}$ from $10^{\circ}$ to $50^{\circ}$ by $20^{\circ}$ with fixed values of $E_{k}=500 \mathrm{MeV}$ and $k_{B} T=200 \mathrm{MeV}$, which are shown in the first column of Figure 2. As $\theta_{p}$ increases, the position of peak is shifted along incoming direction of jet particle rather than outgoing direction of jet particle. And the amplitude of peak is reduced by $\sim 10^{-5}$, roughly when $\theta_{p}$ increases from $10^{\circ}$ to $50^{\circ}$. We can conclude that peak aligns to the incoming jet particle. In the second column of Figure 2, we vary $E_{k}$ from $250 \mathrm{MeV}$ to $750 \mathrm{MeV}$ by $250 \mathrm{MeV}$ with fixed values of $\theta_{p}=10^{\circ}$ and $k_{B} T=200 \mathrm{MeV}$. When $E_{k}$ increases, tails of $d \sigma$ are combined at $\Delta \phi \approx 0$ and possible region of $d \sigma \neq 0$ is getting shrunk. It means that photon with higher energy emits more along the outgoing jet particle. Also we change $k_{B} T$ from $150 \mathrm{MeV}$ to $250 \mathrm{MeV}$ by $50 \mathrm{MeV}$ with fixed values of $\theta_{p}=10^{\circ}$ and $E_{k}=500 \mathrm{MeV}$, whose results are displayed in the third column of Figure 2. As $k_{B} T$ increases, the amplitude of peak increase by $\sim 10$ and the number of collisions between jet particle and medium parton increases due to the active thermal motion of medium partons.

The Figure 3 shows angular correlation between final jet particle and final medium parton for various incident angles of jet particle $\theta_{p}$ and temperatures of system $k_{B} T$. In this correlation, we find that Ridge structure appears at peak and away side. When $\theta_{p}$ increases from $10^{\circ}$ to $50^{\circ}$ by $20^{\circ}$ with fixed $k_{B} T=200 \mathrm{MeV}$, as shown in the first column of Figure 3, the peak-side and away-side Ridge structure moves in the opposite direction to each other. The peak for the medium parton appears almost perpendicular to the pear for the photon because of four-momentum conservation. As $k_{B} T$ increases from $150 \mathrm{MeV}$ to $250 \mathrm{MeV}$ by $50 \mathrm{MeV}$, we can get same results as that between final jet particle and emitted photon.

\section{Conclusion \& Outlooks}

We calculate the angular correlation for final jet particle and emitted photon and that for final jet particle and final medium parton. We check the dependency of the angular correlation for two particles on the incident angle of jet particle, energy of emitted photon and temperature of systems, separately. We set the initial and final energy of incoming jet particle to be $10 \mathrm{GeV}$ and $9 \mathrm{GeV}$. We discover 

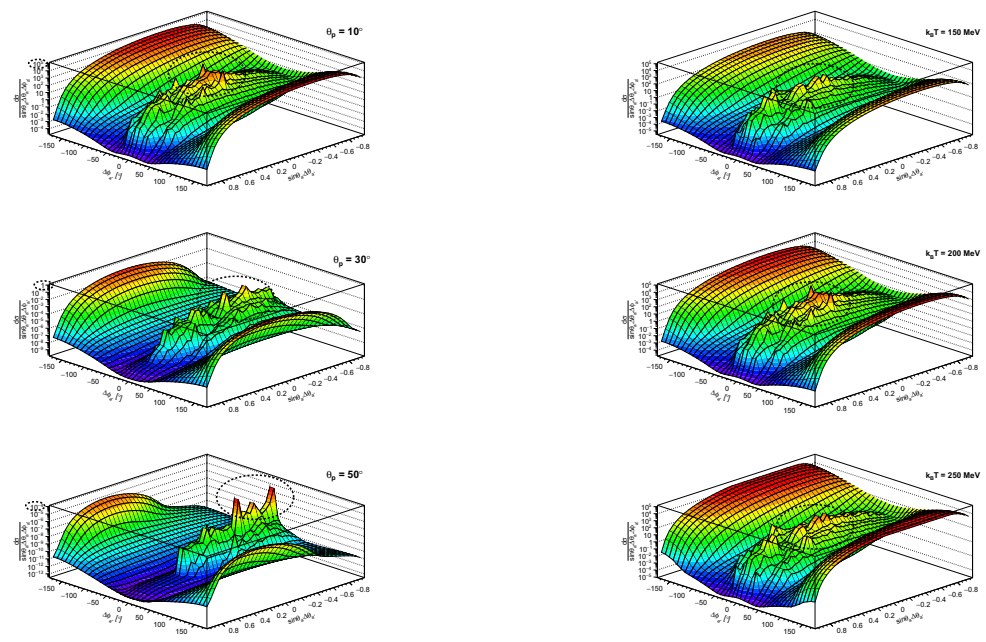

Figure 3. Angular correlation between final jet particle and final medium parton.

collective motion in both angular correlations. The peak aligns to incoming jet particle in correlation between final jet particle and emitted photon. And Ridge structure appears in correlation between final jet particle and final medium parton. From these results, we can conclude that collective motion such as Ridge structure is able to be understood through kinematic interpretation. However, we have to study further effect to fully describe the experimental result. For example, we need to consider boost and flow effects on the momentum distribution for initial medium partons. Also multiple scattering process should be counted. Eventually, we need to include gluon radiation process, which has more important contribution in high-energy heavy-ion collisions than Bremsstrahlung.

\section{Acknowledgements}

This research is funded by Inha University.

\section{References}

[1] C. Y. Wong, Introduction to high-energy heavy-ion collision (World scientific, Singapore, 1994)

[2] C. Itzykson and J-. B. Zuber, Quantum field theory (McGraw-Hill, New York, 1980)

[3] M. E. Peskin and D. V. Schroeder, An introduction to quantum field theory (Westview, USA, 1995)

[4] C. Y. Wong, Physical Review C 85, 064909 (2012)

[5] ATLAS collaboration, Physical Review Letters 116,172301 (2016)

[6] CMS collaboration, Physics Letters B 724, 213240 (2013) 